\title{
Application of Compact Pneumatic Actuators to Laparoscopic Manipulator
}

\author{
Kim Daeyoung ${ }^{1}$, Ryoichi Nakamura ${ }^{2}$, Etsuko Kobayashi ${ }^{1}$, \\ Ichiro Sakuma ${ }^{1}$ and Takeyoshi Dohi ${ }^{1}$ \\ ${ }^{1}$ Institute of Environment Studies, Graduate School of Frontier Sciences, \\ ${ }^{2}$ Department of Precision Machinery Engineering, Graduate School of Engineering \\ The University of Tokyo, 7-3-1 Hongo Bunkyo-Ku, Tokyo, 113-8656, Japan \\ \{young, ryoichi, etsuko, sakuma, dohi \}@miki.pe.u-tokyo.ac.jp
}

\begin{abstract}
In this study, as an alternative actuator for medical robot, pneumatic actuators are applied to laparoscopic manipulator, and their effectiveness was evaluated. Pneumatic actuators have a strong advantage in application to medical systems that the whole mechanism can be sterilized including actuator. We have developed a new laparoscopic manipulator system. Compared with other types of laparoscopic manipulators, it is small and light. The control system can be placed far from the patient by connecting controlling system and the manipulator with air tubes. In conclusion, we confirmed that the range of view and resolution were very useful and acceptable for clinical use and that this paper provides significant first step toward a clean and small medical robot useful in future.
\end{abstract}

\section{Introduction}

There are a growing number of examples of surgical robot systems[1][2]. Repeatable tool position, steady motion, and stability in a fixed position are fundamental advantages of the use of robots. Nevertheless, there are still many problems in motordriven robot. Generally, it is very difficult to sterilize electric motors as a whole and big. Special mechanisms are used to separate sterilized part and non-sterilized part[2], or the total system is covered by sterilized drapes.

Motivated by the need for providing a small and clean robot, we studied the effectiveness of pneumatic actuators to Laparoscopic Manipulator.

\section{System}

At first, we designed the system that can be fixed on the end of steady holder. At the tip of the holder, 2DOFs' joint. The joint (in the circle) has motions of rolling and pitching, so it is possible to rotate the laparoscope. 


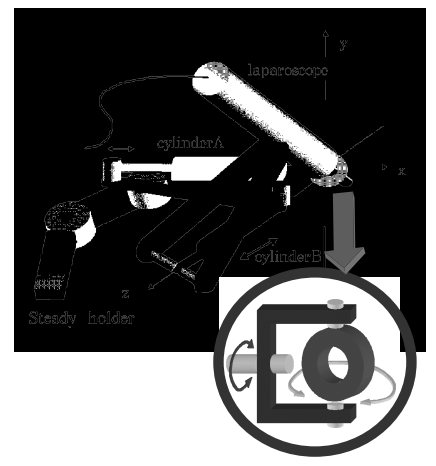

Fig. 1.(a) The concept of the structure (b) Prototype of pneumatic laparoscope system

The cylinder-A controls the horizontal movements, and the cylinder-B controls the vertical movements (Fig.1(a)). By the sliding of the cylinder-A, the laparoscope rotates the $\mathrm{y}$-axis in $\mathrm{xz}$ - plane. When the cylinder-B slides, the laparoscope rotates the $\mathrm{x}$-axis.

\section{Discussion and Conclusion}

In this study, a small and light laparoscopic manipulator was realized by introducing pneumatic linear actuators.

For the size $(20 \mathrm{~cm} \times 10 \mathrm{~cm} \times 10 \mathrm{~cm})$, it makes possible to reduce the space could be obstruct. About the view range, we knew $38^{\circ}$ in horizontal and $18^{\circ}$ in vertical range that it could see the whole of the liver model when it is far from $20 \mathrm{~cm}$ from the tip of laparoscope. The resolution less than $0.5 \mathrm{~mm}$ in cylinder, corresponding to $0.3^{\circ}$ in angle of view.

Simple structure and connecting mechanism made it easy to assemble the system after sterilization since the whole mechanism can be sterilized including actuator.

We confirmed the effectiveness of pneumatic actuator in designing clean and compact medical robots.

This study was partly supported by the Research for the Future Program (JSPS-RFTF 99100904).

\section{References}

1. Taylor RH, Funda J, Eldridge B, Gomory S, Gruben K, LaRose D, Talamini M, Kavossi L, Anderson J A Telerobotic Assistant for Laparoscopic Surgery. Computer Integrated Surgery: The MIT Press, (1995) pp 581-592

2.Etsuko Kobayashi 5-links laparoscope manipulator for minimally invasive operation, 1997 